\title{
Resolution of the resolution limit.
}

\section{James M. Holton}

Department of Biochemistry and Biophysics, University of California San Francisco

Division of Molecular Biophysics and Integrated Bioimaging, Lawrence Berkeley National Lab

Structural Molecular Biology Group, Stanford Synchrotron Radiation Lab, SLAC

JMHolton@lbl.gov

There has been much debate over resolution limits of late, and an objective re-visiting of what resolution means and why we apply them is warranted.

The reason for cutting off diffraction data beyond a certain "resolution limit" is to prevent adding unnecessary noise into the refinement and final electron density map. But, on the other hand, cutting off the data too harshly leads to series-termination errors that also corrupt the map. There must be some "happy medium" cutoff where the total error is minimized. I will present a simple theoretical calculation that shows this optimum resolution cutoff lies where the popular $\mathrm{CC}_{1 / 2}$ statistic drops to zero. At this point $\mathrm{CC}^{*}$ is numerically unstable, making the optimum cutoff difficult to assess in practice. Fortunately, the noise introduced by an over-optimistic resolution cutoff is small, and may be effectively eliminated by replacing the resolution cutoff with a rolloff that simply requires that the Wilson plot of the data forms a straight line beyond the traditional "resolution limit". With this filter all measured spots can be used with impunity, and the optimum resolution cutoff becomes $0.0 \AA$.

This modification is an extreme example of the disparity between the resolution cutoff of the data used in refinement and the effective resolution of the final model, which is really the "resolution" of interest. To evaluate this, we turn to the traditional definition of "resolution" from optics, which is the minimum distance between two atoms that still allows them to be "resolved" or identified as two peaks rather than one. This distance is directly related to the atomic B factors, and is improved by sharpening. The "true" resolution is therefore the point where the noise in the electron density map is amplified by the sharpening enough to compromise the distinguishing one peak from two. This can be assessed empirically using the RAPID procedure (Lang et al. 2014). Since the dominant source of error in electron density maps is the discrepancy between $F_{\text {obs }}$ and $F_{\text {calc }}$, the $R_{\text {work }} / R_{\text {free }}$ factors can be made to play a role in a consistent re-assessment of the true resolution of all models deposited in the Protein Data Bank. 